\title{
ARTICLE
}

\section{DEPENDENCE OF THE CUT-OFF PARAMETERS FOR THE CLUSTER RECONSTRUCTION ON INCIDENT MOMENTA}

\author{
Shinebayar J. ${ }^{1 *}$, Togoo R. ${ }^{2}$, Tursukh . $^{2}$, Otgonsuren $D .^{2}$ and Munkhjargal L. ${ }^{2}$ \\ ${ }^{l}$ Department of Lifelong Education and E-learning, \\ Mongolian National University of Education, Ulaanbaatar, Mongolia \\ ${ }^{2}$ Department of Elementary Particles and Nuclear Physics, Institute of Physics and Technology, \\ Mongolian Academy of Sciences, Ulaanbaatar, Mongolia
}

ARTICLE INFO: Received: 19 Oct, 2018; Accepted: 18 Dec, 2018

\begin{abstract}
In this work, firstly, we have determined the multiplicities of protons that are involved from projectiles to interactions, and secondly, the mean incident momentum using experimental data on the collisions of proton, helium, oxygen, silicon, and sulfur with the nuclear emulsion at $4.5 \mathrm{~A} \mathrm{GeV/c}$. We have studied the dependences of clot numbers, number of particles in the clot, rapidity, and the mean azimuthal value on the variable $d_{i j}$, and the dependence of the cluster number on the mean value of the variable $d_{i j}$ " Using the "Cone" algorithm for the clustering, we have precisely identified that the cut-off parameter to compose the clusters consisted of s and f tracks produced in ( $p, \mathrm{He}, \mathrm{C}, \mathrm{O}, \mathrm{Si}, \mathrm{S})+$ Em collisions decreases by the power law with mean values of incident momentum.
\end{abstract}

Keywords: Collision; high-energy; particle; nucleus; rapidity; scattering and azimuthal angles; multiplicity; nuclear photographic emulsion; momentum; cluster; cut-off parameter; algorithm;

\section{INTRODUCTION}

The clusterization of secondary particles had been reconstructed by non-invariant quantities in the mid-1970s [8][11] and by invariant quantities in the mid-1980s [3][5] until it was reconstructed by $\mathrm{k}_{\mathrm{T}}$ algorithm in the beginning of the 1990s [9]. We have done our research of the clusterization of particles by this $\mathrm{k}_{\mathrm{T}}$ algorithm [6][15]. With the photoemulsion experimental data, the dependence of the incident momentum of the cut-off parameter upon the type of colliding objects needs to be further studied.

A nuclear photo-emulsion medium consists of a varieties of nucleus, like $\mathrm{H}, \mathrm{C}, \mathrm{N}, \mathrm{O}, \mathrm{Ag}$ and $\mathrm{Br}$. There are different types of nuclear emulsion having different composition of nucleus. BR-2 nuclear emulsions with consideration of $1 \mathrm{~cm}^{3} \cdot 10^{-22}$ nuclear volume, is composed of $3.15(\mathrm{H}), 1.41(\mathrm{C}), 0.395(\mathrm{~N})$, 0.956(O), 1.0331(Br) ба 1.031(Ag). We have determined the parameters for the cluster reconstruction basing on the data in nucleus- 
nucleus collisions using nuclear emulsion track detector [4] [12-13] [18] and defined the cut-off parameters for the cluster by the experimental data, which were produced at

\section{MATERIALS AND METHODS}

Stacks of Br-2 nuclear emulsions were exposed to a $4.5 \mathrm{~A} \mathrm{GeV} / \mathrm{c}^{16} \mathrm{O}$ beam at the Synchrophasotron, Dubna. The stacks are consisted of 50 or 100 pellicles with a dimension of $20 \mathrm{~cm} \times 10 \mathrm{~cm} \times 600 \mu \mathrm{m}$ (undeveloped emulsion). The intensity of irradiation was $10^{4}$ particles $/ \mathrm{cm}^{2}$, and the beam diameter was about $1 \mathrm{~cm}$. Along-the-track, double scanning was carried out, fast in the forward direction and slow in the backward direction. The scanned beam tracks were further examined by measuring the delta-electron density on each of them to exclude the tracks having a charge smaller than the beam-particle charge.

One-prong events with a secondaryparticle track emission with an angle of less than three degrees and without visible tracks from excitation or disintegration of the target nucleus were excluded due to the elastic scattering. Experimental details have been published [9] [11], [16-17].

In the present study, nuclear emulsion track detector has been used. Nuclear emulsion detectors offer a good angular resolution $(\sim 0.1$ $\mathrm{mrad})$ and they are capable of registering all the produced particles in the $4 \pi$ geometry [14]. The detector is mostly used to record and permanently store the information of the charged particles with the different ionizing power.

In the measured events, the secondary particles are classified as follows:

- black particle tracks (b) having a range $L<3 \mathrm{~mm}$ in emulsion which corresponds to a proton kinetic energy of $<26 \mathrm{MeV}$;

- grey particle tracks (g) having relative ionization $I^{*}\left(=I / I_{0}\right)>l .4$ and $L>3 \mathrm{~mm}$ which corresponds to a proton kinetic energy of $26-400 \mathrm{MeV}$, where $I$ is the the Dubna Propane bubble chamber [17]. The cut-off parameters for the clusters in these collisions are very important for studying resonance production.

particle track ionization and $I_{0}$ is the ionization of a shower track in the narrow forward cone of an opening angle of $3^{\circ}$;

- the $b$ and/or $g$ particle tracks are called heavy-ionizing-particle tracks (h);

- shower particles (s) having $I^{*} \leq 1.4$. Tracks of this type with an emission angle of $<5^{\circ}$ were further subjected to rigorous multiple scattering measurement for momentum determination and, consequently, for separating the produced pions and the single charged projectile fragments (PF) (i.e. protons, deuterons and tritons). The ratio of ${ }^{1} \mathrm{H}:{ }^{2} \mathrm{H}:{ }^{3} \mathrm{H}$ was found to be 63:27:10 [13]. Furthermore, the $\mathrm{Z}=1 \mathrm{PF}$ are not included in the $s$ particles.

- The multicharged $Z>2$ PF are subdivided into $Z=2,3, \ldots, 16$ fragments according to the measured delta-electron and/or the gap density. Thus, all particles were adequately divided into $\mathrm{PF}$ of $\mathrm{Z}=1-16$, target fragments (TF), i.e. h particles, and the generated s particles. The total charge of the $P F, Z^{*}=\sum n_{i} Z_{i}$, was calculated in each star, where $n_{i}$ is the number of fragments of charge $Z_{i}$ in an event.

For each track we obtained from measurements: (a) the polar angle $\vartheta$, i.e. the space angle between the direction of the beam and that of the given track, and (b) the azimuthal angle $\varphi$, i.e. the angle between the projection of the given track in the plane normal to the beam and the direction perpendicular to the beam in this plane (in an anticlockwise direction). The polar $(\vartheta)$ and the azimuthal $(\varphi)$ emission angles of all tracks were measured. The pseudorapidity $\eta=-\ln [\tan (\vartheta / 2)]$ was calculated for each relativistic particle. 
For the (p, He, C, O, Si, S) + Em interactions detected by the nuclear emulsion track detector, the specific cut-off parameter in the chosen space should be determined for distinguishing the clusters reconstructed with the $\mathrm{s}$ and the $\mathrm{f}$ particles. We have chosen the two invariant quantities - the azimuthal angle $(\varphi)$ and the pseudorapidity $(\eta)$ of secondary particles produced by the interactions as mentioned above and have used the cone algorithm for distinguishing the clusters [10]. The cone algorithm is a specific type of $\mathrm{k}_{\mathrm{T}}$ algorithm. The idea is as follows: $d_{i j}=R_{i j}^{2}$ becomes easier when we set $p$ parameter to be 0 and $D_{n}$ to be 1 for the equation $d_{i j}=\min \left\{p_{T, i}^{2 p}, p_{T, j}^{2 p}\right\} \cdot R_{i j}^{2} / D_{0}^{2}$ of $\mathrm{k}_{\mathrm{T}}$ algorithm. Since the momentum of secondary particles produced by these interactions can't be measured, we set $\mathrm{p}$ parameter to be 0 [9].

Firstly, for the secondary particle pairs in the (p, He, C, O, Si, S) + Em interactions detected by the nuclear emulsion track detector, we have studied the dependence of all possible minimum and maximum values of the variable $d_{i j}$ on random numbers that contain specific number clusters and showed it in figure 1. For example, in figure 1, the top left figure, the dependence of cluster numbers $\left(N_{c l}=0,1,2,3\right)$ on the values of $d_{i j}$ is shown for the cluster forming process for the relative $\mathrm{s}$ particle and the $\mathrm{f}$ fragments of the beam nucleus produced by the proton and the photoemulsion interaction.

It should be noted that it's impossible to determine the rapidities of black and grey tracks with the ionization of secondary particles, and therefore, we have not included these particles in our research.

Secondly, we have defined the mean value $<d_{i j}>$ of the variable $d_{i j}$ from the dependence as shown in the figure 1 and showed the dependence of the cluster numbers $N_{c l}$ on variables of $d_{i j}$ in figure 2. The latter dependence is approximated with the sum of two exponent functions [17]. The values of the coefficients defined by approximation are shown in columns 6 and 7, and the values of $\chi^{2}$, the number of degrees of freedom, are shown in column 8 . In figure 2 , the red vertical line represents the position of the cut-off parameter $\mathrm{d}_{\text {cut }}$, and this red line denotes the intersections of the approximated lines near $<d_{i j}>\sim 7.1$ for the p+Em interaction, $<d_{i j}>\sim 6.0$ for the $\mathrm{He}+\mathrm{Em}$ interaction, $<d_{i j}>\sim 5.0$ for the $\mathrm{C}+$ Em interaction, $<d_{i j}>\sim 3.8$ for the $\mathrm{O}+$ Em interaction, $<d_{i j}>\sim 3.6$ for the $\mathrm{Si}+\mathrm{Em}$ interaction, and $\left\langle d_{i j}>\sim 3.2\right.$ for $\mathrm{Si}+\mathrm{Em}$ interaction correspondingly. These values are shown in column 5 of table 1 .

To prove that the cut-off parameters we have defined are real, we have studied the clot numbers, numbers of secondary particles in the clot, their rapidities, and the mean value of the azimuthal angle, and we have shown the dependence of these mean values on $d_{i j}$ with their interactions, in figure 3. Similarly like figure 2, the red vertical lines represent the cut-off parameters, and it appears that the curves, on the right side of these lines, could be approximated with a uniform function. For example, for the S+Em interaction in the right bottom most side of figure 3 , it was observed that the mean value of the clot numbers decreases exponentially, the mean value of charged particles in the clot rises exponentially, and the mean rapidity and the azimuthal angle are almost constant. On the other hand, the curves, on the left side of the cut-off lines, could be risen or dropped with a non-uniform function.

We have determined the mean momentum $\left(<p_{0}>\right)$ of the primary nucleus used for this interaction when we excluded the sum of the charges of the particles, which haven't been involved in the interaction, from the primary nuclear charge and defined the mean value $\left(<v_{p}>\right)$ of protons, which have been involved in the collision, from the beam nucleus, and multiplied the mean value with $4.5 \mathrm{GeV} / \mathrm{c}$ as listed in column 3 and 4 . Also in the bottom most row of the table, the quantities of the types of interactions, the statistics of events, 
and the momentum per nucleus correspond to the oxygen-photo emulsion interaction at 60 $\mathrm{GeV} / \mathrm{c}$.

In figure 4 , the dependence of $d_{\text {cut }}$ parameter, forming the cluster with the $\mathrm{s}$ and the $\mathrm{f}$ particles, on the mean momentum $\left\langle p_{0}>\right.$ is shown. From this, it is observed that this dependence is falling with the power law.

Table 1. The values of event number, the mean multiplicities of interacting protons, their average momentum consumed in the interactions, the cut-off parameter in the nuclear-nuclear collisions, and the fitting parameters.

\begin{tabular}{|c|c|c|c|c|c|c|c|}
\hline $\begin{array}{c}\text { Interac- } \\
\text { tion type }\end{array}$ & $\begin{array}{c}\text { Event } \\
\text { number }\end{array}$ & $\left\langle v_{\mathbf{p}}>\right.$ & $\left\langle\boldsymbol{p}_{\mathbf{0}}>, \mathbf{G e B C} / \mathbf{c}\right.$ & $\boldsymbol{d}_{\text {cut }}$ & $\alpha$ & $\beta$ & $\chi^{2} / \mathbf{N D F}$ \\
\hline $\mathrm{p}$ & 2576 & $1.0 \pm 0.02$ & $4.5 \pm 0.1$ & $7.1 \pm 2.0$ & $0.51 \pm 0.18$ & $1.38 \pm 0.21$ & $0.0 / 1$ \\
& 977 & $1.23 \pm 0.04$ & $5.62 \pm 0.15$ & $6.0 \pm 0.7$ & $0.95 \pm 0.29$ & $1.82 \pm 0.87$ & $0.1 / 1$ \\
& 1931 & $2.69 \pm 0.06$ & $12.10 \pm 0.40$ & $5.0 \pm 0.5$ & $0.63 \pm 0.13$ & $2.26 \pm 0.69$ & $1.5 / 6$ \\
& 2823 & $3.15 \pm 0.06$ & $14.17 \pm 0.27$ & $3.8 \pm 0.4$ & $0.77 \pm 0.09$ & $2.61 \pm 0.37$ & $11.8 / 11$ \\
& 1318 & $4.49 \pm 0.12$ & $20.20 \pm 0.54$ & $3.6 \pm 0.4$ & $0.87 \pm 0.09$ & $3.2 \pm 0.8$ & $21.2 / 15$ \\
& 1322 & $4.78 \pm 0.13$ & $21.51 \pm 0.58$ & $3.2 \pm 0.4$ & $0.69 \pm 0.08$ & $3.51 \pm 0.78$ & $12.9 / 11$ \\
\hline$*$ & 834 & $4.61 \pm 0.09$ & $276.6 \pm 5.4$ & $2.0 \pm 0.01$ & $0.66 \pm 0.14$ & $0.13 \pm 0.07$ & $6.5 / 25$ \\
\hline
\end{tabular}

* In this row, we have illustrated the corresponding values calculated from the oxygen-emulsion interactions at $60 \mathrm{GeV/c}$ per nucleon. 

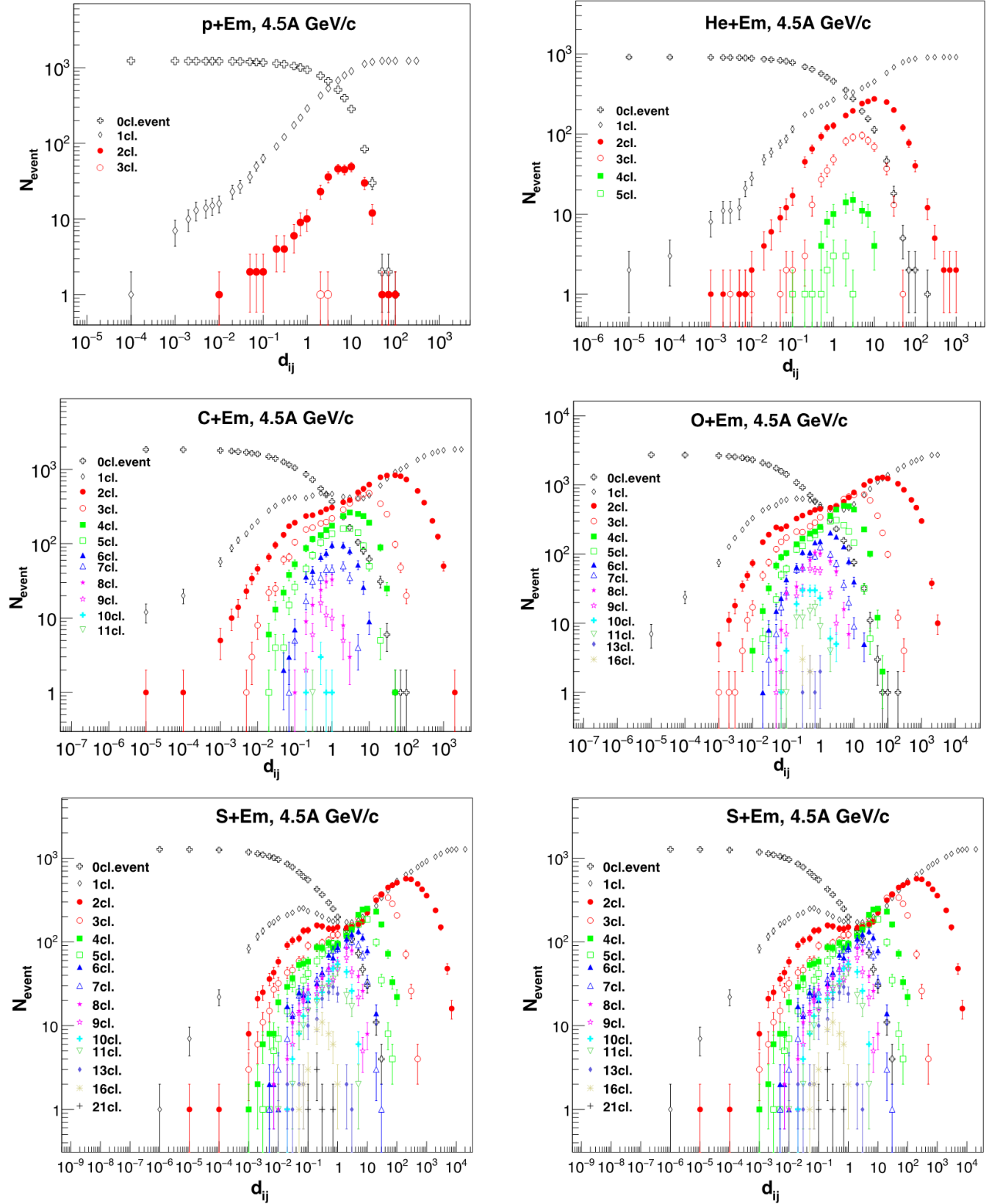

Figure 1. Dependence of the events number $N_{\text {event }}$ with the clusters on the variable $d_{i j}$ These figures are taken into account for the number cluster 0, 1, 2, 3 in p Emat 4.5AGeV/c (in the top left figure), the number cluster 0, 1, 2, .., 5 in He+Em at 4.5A GeV/c (in the top right figure), the number cluster 0, 1, 2, ..., 11 in $\mathrm{C}+\mathrm{Em}$ at 4.5A GeV/c (in the center left figure), the number cluster 0, 1, 2, ..., 16 in $\mathrm{O}+\mathrm{Em}$ at $4.5 \mathrm{~A} \mathrm{GeV/c}$ (in the center right figure), the number cluster 0, 1, 2, .., 20 in Si+Em at 4.5A GeV/c (in the bottom left figure), the number cluster 0, 1, 2, .., 21 in S+Em at 4.5A GeV/c (in the bottom left figure) interactions. 

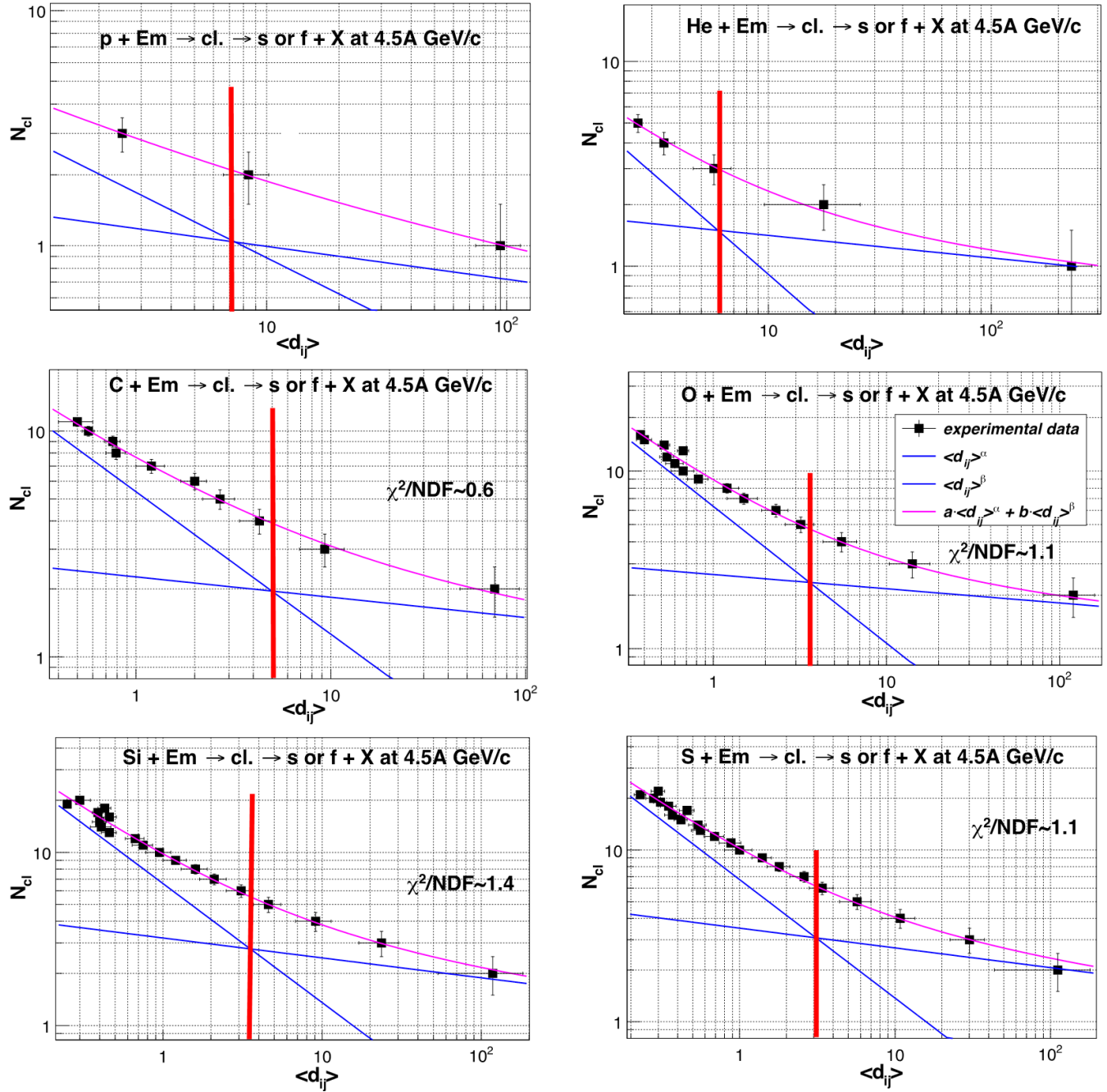

Figure 2. Dependence of the mean value $<d_{i j}>$ of the variable $d_{i j}$ on cluster number $N_{c r}$. The two blue lines are calculated by the sum of two power functions. The red vertical line represents the intersect of the two blue lines. The pink curve represents the fit results. The interactions type was presented on the top of each figure. 

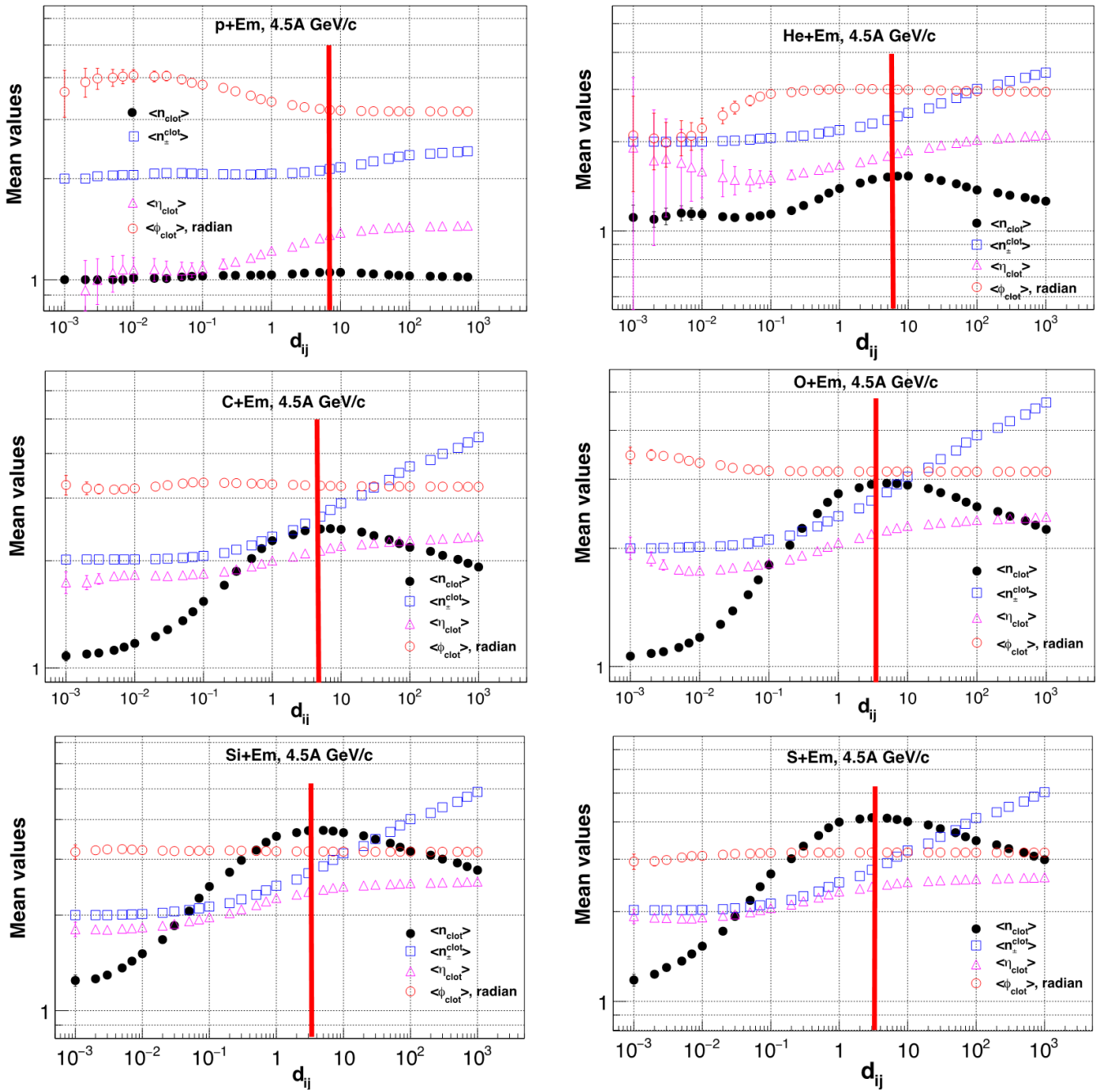

Figure 3. Dependences of the clot numbers, number of particles in the clot, the rapidity, and the average azimuthal value on the variable $d_{i j}$. The red vertical line represents the value of cut-off parameter in each collision. And the interaction type was presented on the top of each figure. 


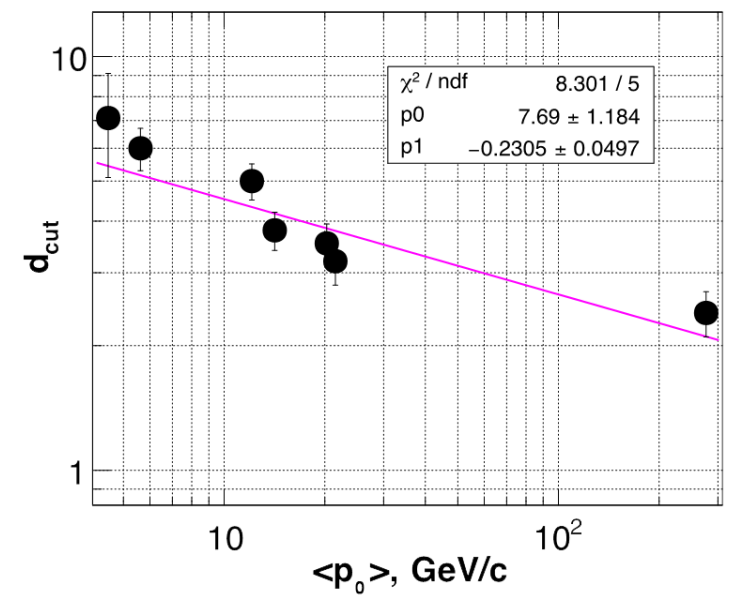

Figure 4. Dependence of the cluster number on the average value of the variable $d_{i j}$. The pink curve represents the fit results.

\section{CONCLUSIONS}

1. The experimental data has been taken in interactions of proton, helium, carbon, oxygen, silicon, and sulfur with the nuclear emulsion in this research.

2. Firstly, we have determined the multiplicities of protons that were involved from projectile to interactions, and secondly, the mean momentum consumed in the interactions using the experimental data on collisions of the ( $\mathrm{p}, \mathrm{He}, \mathrm{C}, \mathrm{O}, \mathrm{Si}$, S) + Em.

3. Using the "Cone" algorithm for clustering, we have precisely identified that the cut-off parameter to compose the clusters consisted of $\mathrm{s}$ and $\mathrm{f}$ tracks produced in the (p, He, C, $\mathrm{O}, \mathrm{Si}, \mathrm{S})+$ Em collisions decreases by the power law with average values of incident momentum.

Acknowledgement: The authors are grateful to Nuclear photo emulsion colleagues in JINR, Dubna, Russia who exposed and processed the emulsion plates, and extracted the experimental data for this analysis.

Also, we would like to thank the Ministry of Education, Culture, Science and Sports of Mongolia for its financial support through the research project No.ShuSS-2018/27.

\section{REFERENCES}

[1] Ameeva, B.U., et al, 1986, Izv. Akad. Nauk. 112703.

[2] Andreeva, N.P., et al, 1986, JINR Preprint, Dubna P1-86-8.

[3] N. Angelov, V. B. Lyubimov, R. Togoo (Dubna, JINR), Clusterization in processes of multiple particle production by nuclei and its connection with cumulative creation of hadrons (in Russian), 1989, Published in Dubna JINR - 5(38)-89 (89,rec.Feb.90) 1125.

[4] V. Anzon, I. Ya. Chasnikov, "High Energy Physics and Cosmic Ray", "Nauka”, AlmaAta, 1974, p. 23.

[5] Baldin, A. M. et al, Universal Four-dimensional Hadron Jets And The Observability of Color Charges, JINR-E1-87-142.

[6] Bannik, B.P., et al, 1985 Z. Phys. A, 321, 249. 
[7] Bannik, B.P., et al, 1984, Zh. Eksp. Teor. Fiz. Pis. Red. 39184 (1984 JETP Lett. 39 219) [24] Vokalova, A., et al, 1984, JINR Communication, Dubna P1-84-532.

[8] J. D. Bjorken and S. J. Brodsky, Statistical Model for Electron-Positron Annihilation into Hadrons, Phys. Rev. D1, 1416, 1970.

[9] S. Catani (CERN), Yuri L. Dokshitzer (St. Petersburg, INP \& Lund U.), M. H. Seymour (Lund U.), B. R. Webber (CERN), Longitudinally invariant $\mathrm{k} \perp$ clustering algorithms for hadron collisions, Feb 1993, p. 37,Nucl.Phys. B406(1993) 187-224DOI:10.1016/05503213(93)90166-M CERN-TH-6775-93, LU-TP-93-2.

[10] Dorschitzer, Yuri L., Leder, G. D., Moretti and Webber B.R., (1997), Better jet clustering algorithms. 2HEP, 08, 001, doi:10.1088/1126-6708/1997/08/011

[11] G. Hanson et al. [SLAC-LBL Collaboration], Evidence for Jet Structure in Hadron Production by e+ e- Annihilation, Phys. Rev. Lett.35, 1609 (1975); Hadron Production 67 by e+e - Annihilation at Center-of-Mass Energies between $2.6 \mathrm{GeV}$ and $7.8 \mathrm{GeV}$, Part 2: Jet Structure and Related Inclusive Distributions, Phys. Rev. D26, 991, 1982.

[12] E. Lohrmann and M. W.,Tevcher. Nuovo Cimento, 25, 957, 1962.

[13] N. V. Maslennikova., et al, Proc. of the 8th Intern. Conf. on Nuclear photographic and solid state track detectors, p.132, Bucharest. 1972.

[14] Perfilov N. A., Lozhkin O.V. and Gorichev P.A., 1960, Usp. Fiz. Nauk 703.

[15] Shinebayar, J., Sovd, M., Togoo, R., Tulgaa, T., Tursukh, A., Proceedings of the Mongolian academy of Sciences, Vol. 58, No.1 (225), Ulaanbaatar, 2018. DOI: https:// doi.org/10.5564/pmas.v58i1.973

[16] K. D. Tolstov et al, JINR, P1-8313, Dubna, 1974.

[17] Togoo, R., Otgonsuren, D., Shinebayar, J., Proceedings of the Mongolian academy of Sciences, Vol. 52, No.2 (210), Ulaanbaatar, 2014. DOI: http://dx.doi.org/10.5564/ pmas.v54i 2.657

[18] A. O. Weisenberg, et al., YadFiz, 18, 1239, 1973. 